AperTO - Archivio Istituzionale Open Access dell'Università di Torino

The JEM-EUSO observation in cloudy conditions

This is a pre print version of the following article:

Original Citation:

Availability:

This version is available http://hdl.handle.net/2318/1636723

since 2017-05-19T18:41:56Z

Published version:

DOI:10.1007/s10686-014-9377-2

Terms of use:

Open Access

Anyone can freely access the full text of works made available as "Open Access". Works made available under a Creative Commons license can be used according to the terms and conditions of said license. Use of all other works requires consent of the right holder (author or publisher) if not exempted from copyright protection by the applicable law. 
Noname manuscript No.

(will be inserted by the editor)

\title{
1 JEM-EUSO observation in cloudy conditions
}

\author{
2 JEM-EUSO Collaboration
}

4 Received: date / Accepted: date
Alejandro Guzmán

Institute for Astronomy and Astrophysics, Kepler Center, University of Tübingen, Sand 1, D-72076

Tübingen, Germany

E-mail: guzman@astro.uni-tuebingen.de

Guadalupe Sáez Cano

SPace and AStroparticle Group, Universidad de Alcalá, Ctra. Madrid-Barcelona km 33.7, E-28871 Alcalá de Henares, Spain

E-mail: lupe.saez@uah.es

Kenji Shinozaki

Institute for Astronomy and Astrophysics, Kepler Center, University of Tübingen, Sand 1, D-72076

Tübingen, Germany

Tel.: +49-7071-29 25279

Fax: +49-7071-29 3458

E-mail: shinozaki@astro.uni-tuebingen.de 


\section{Introduction}

The space-based extensive air shower (EAS) observation, as employed in the JEMEUSO (Extreme Universe Space Observatory on-board the Japanese Experiment Module) mission [1-5], is a novel approach for investigating ultra-high energy cosmic rays (UHECRs; referred to as $\gtrsim 5 \times 10^{19} \mathrm{eV}$ ). The fluorescence technique is applied to search for the moving track of ultra-violet (UV) photons produced in EAS development in the nighttime atmosphere. This technique has been established by the ground-based experiments [6] but has never been put into practice in space, thus requiring specific considerations. In the present article, we discuss characteristics of EAS observed in different atmospheric conditions by the JEM-EUSO mission, focusing on the role of clouds.

The JEM-EUSO observatory is an ensemble of the UV telescope, referred to as 'main telescope', the atmospheric monitoring (AM) system [7,8], and other subsystem instruments. It is designed to operate on the JEM Kibo module of the International Space Station (ISS) [9,10]. Orbiting at a nominal altitude $H_{0} \sim 400 \mathrm{~km}$ from the Earth's surface ${ }^{1}$, it revolves every $\sim 90$ min at a speed of $\sim 7.6 \mathrm{~km} \mathrm{~s}^{-1}$. On average, the ISS spends $\sim 34 \%$ of the time in umbra of the Earth, during which the EAS observation may be conducted. Accounting for the effect due to back-scattered moonlight, the EAS observation duty cycle is expected to be $\sim 20 \%$ [11]. According to the inclination, the ISS operation ranges between the latitudes $\pm 51.6^{\circ}$.

The main telescope is designed to have a wide field-of-view (FOV), covering an area of $\sim 1.4 \times 10^{5} \mathrm{~km}^{2}$ in nadir observation. It consists of a $4.5-\mathrm{m}^{2}$ refractive optics and a focal surface (FS) detector. The FS detector is formed by 137 photo-detector modules (PDMs) [4,12]. Each PDM is a set of 36 multi-anode photomultiplier tubes (MAPMTs) having 64 pixels with a spatial window of $0.075^{\circ}$ equivalent to $\sim 0.5 \mathrm{~km}$ on the Earth's surface. The integration time of data acquisition is $2.5 \mu \mathrm{s}$ and is called gate time unit (GTU). Two levels of trigger algorithms [13] search every PDM for stationary and transient excesses of EAS signals against prevailing background light.

The AM system consists of an infra-red (IR) camera [14,15] and a steerable UV laser system [7,8]. To characterize the cloud distribution, the IR camera measures the brightness temperature distribution over the FOV of the main telescope. This provides the relative distribution of the cloud-top altitude in the FOV. The laser system with the main telescope acting as a receiver allows a LIDAR (light detection and ranging) technique to sound the atmospheric properties along the line of sight of interest. LIDAR information is used to calibrate the brightness temperatures with the absolute altitude. Clouds with small optical depths may be observed with temperatures that do not correspond to the actual altitudes. In this case, LIDAR information that aims to distinguish between clear atmosphere and clouds with given thresholds in optical depth may label the EAS events taking place in such regions. For details regarding instrument, operation, data treatment, etc. of the AM system, see Ref. [7,8,16,17].

In the following sections, we estimate the efficiency of the EAS observation in atmospheric conditions, with and without clouds, using dedicated simulation studies

\footnotetext{
${ }^{1}$ Hereafter, Earth's surface is referred to as the assumed Earth's ellipsoid model and the altitude is measured from this level.
} 
for the JEM-EUSO mission. We also analyze the cloud coverage using available databases from meteorological missions. Combining both factors, we estimate the overall observation efficiency with a perspective towards event reconstruction.

\section{EAS observable properties and efficiency of trigger under cloudy conditions}

In UHECR observation by optical means, isotropically emitted fluorescence light is the dominant component of the signals and its luminosity is almost proportional to the energy deposited by the EAS particles. Highly beamed Cherenkov light is also produced close to the particle trajectory. A part of this light may reach the JEMEUSO telescope once it is scattered in the atmosphere towards the direction of the telescope. In addition, the space-based observation also detects the diffusely reflected Cherenkov photons from land or water. A similar effect takes place at the impact of photons on cloud. Those reflected signals, referred to as 'Cherenkov footprint', provide a piece of information on the position and timing of the EAS reaching such boundaries. The geometrical configuration constrains uncertainty in distance to the EAS, as well. In general, spaced-based fluorescence observation favors EASs from large zenith angles with little effect of aerosols near the Earth's surface. These points simplify full calorimetric measurement of the development of EAS.

In actual observation, ground-based observatories are affected by local weather conditions. As far as the influence of clouds is concerned, the EAS observation can be performed without further consideration by selecting times without cloud coverage. In this case, the exposure is only lowered by the reduction of observation time. On the other hand, space-based telescopes overlook continuously changing landscapes within their wide FOV. The atmospheric conditions are also largely variable by location and time along the satellite trajectory. This leads the JEM-EUSO telescope to watch all possible conditions, in particular presence of clouds in the FOV. The timescale of transitions between cloudy and clear atmosphere conditions may be an order of minute or shorter. Seasonal variations also appear every $\sim 20 \mathrm{~min}$, namely quarter of the orbital period. However, the presence of clouds is only relevant if the EAS takes place behind the cloud, especially those with large optical depths. The influence of the cloud is obviously dependent on their top altitude. Therefore, the portion of FOV where high-altitude clouds exist may reduce the instantaneous aperture of EAS observation, while it is possible to detect EAS events within the remaining portion.

The observed temporal and topological profiles of the signals are used to retrieve the geometry and longitudinal development of the EAS (see Refs. [18-20] for details about technique and performances). In practice, the so-called shower-detector-plane (SDP), the plane containing the EAS track and the detector, is determined by orientation of the signals projected on the FS detector. The apparent angular velocity of the light spot indicates the incident direction of the EAS within SDP, presuming that it moves with the EAS at the speed of light. Cherenkov footprint or other methods [3, 19] can be used to determine the distance to the EAS. Knowing the EAS geometry and taking into account extinction loss, the arrival time distribution of photons, namely light curve, may be converted to the energy deposition profile along the EAS. Photons from EAS, heading towards the JEM-EUSO telescope, pass on or near SDP. 

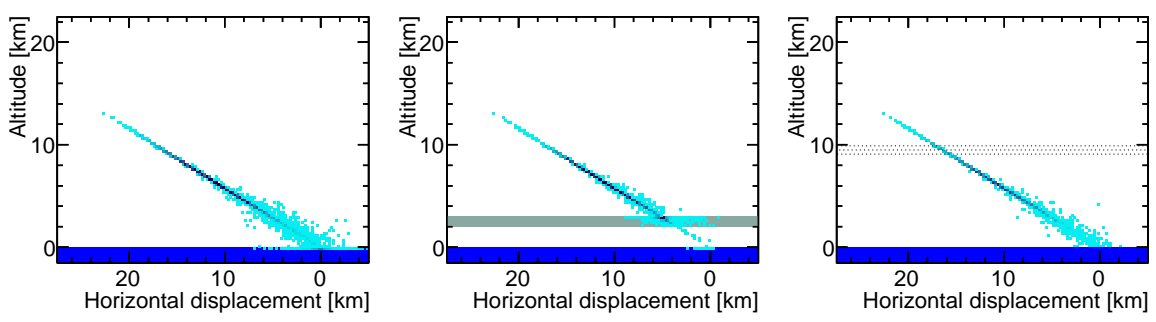

Fig. 1 Schematic view of EAS geometry for $\Theta=60^{\circ}$ in the different atmospheric conditions. The left panel shows the observed photon distribution projected on SDP for the clear atmosphere condition. The middle and right panels are same but for the cloudy cases of large optical depth at $3 \mathrm{~km}$ and of small optical depth at $10 \mathrm{~km}$, respectively.

If a cloud is located between EAS and the detector on that plane, the apparent profile of the signals is affected.

In Fig. 1, a schematic view of EAS geometry is illustrated in the different atmospheric conditions. The left panel shows the observed photon distribution projected on SDP for the clear atmosphere condition. The middle and right panels are the same except for the cloudy cases of large optical depth at $3 \mathrm{~km}$, and of small optical depth at $10 \mathrm{~km}$, respectively. In this example, the zenith angle $\Theta$ of the EAS is $60^{\circ}$.

In the clear atmosphere condition, provided that a bright enough portion of the EAS is contained within the wide-FOV, our space-based telescope is capable of detecting said EAS. Moreover, in many of the cases this entire portion of EAS can be followed until its impact on the Earth's surface.

In order to investigate such effects, we employ ESAF (EUSO Simulation and Analysis Framework) [21]. In the ESAF version used in the present work, the JEMEUSO configuration is implemented [11]. The primary UHECR is assumed to be protons. In addition to the clear atmosphere condition, we simulate EASs through a homogeneous-layer test cloud, with a given cloud-top altitude $H_{\mathrm{C}}$ and optical depth $\tau_{\mathrm{C}}$. Unless otherwise noted, $\tau_{\mathrm{C}}$ hereafter means the vertical optical depth of the cloud components. In the setup of ESAF, two models of the phase function for photon scattering, namely cumulus- [22] and cirrostratus- [23] models, are available to simulate this process. In practice, these models represent the cases for clouds formed by water droplets and ice crystals depending on altitude, respectively. As the scope of the present article is the impact of the cloud on the trigger exposure, the photon intensity at the telescope pupil is more relevant. In this sense, the optical depth is the key parameter for determining such value. In our simulation, the former model is chosen, however, and the effective difference between these models is only apparent in small scattering angles within $\sim 10^{\circ}$. Such difference may be important in the case that, unlikely for spaced-based observation, the telescope may see the direct Cherenkov photons.

In Fig. 2, the top panel shows the light curves of a typical EAS in different atmospheric conditions. The sample is the case for the EAS of $E=10^{20} \mathrm{eV}$ from $\Theta=60^{\circ}$. The solid line represents the case for the clear atmosphere. Dashed and dotted lines denote the cases for clouds of $\tau_{\mathrm{C}}=1$ at $H_{\mathrm{C}}=3 \mathrm{~km}$ and of $\tau_{\mathrm{C}}=0.5$ at $H_{\mathrm{C}}=10 \mathrm{~km}$, 

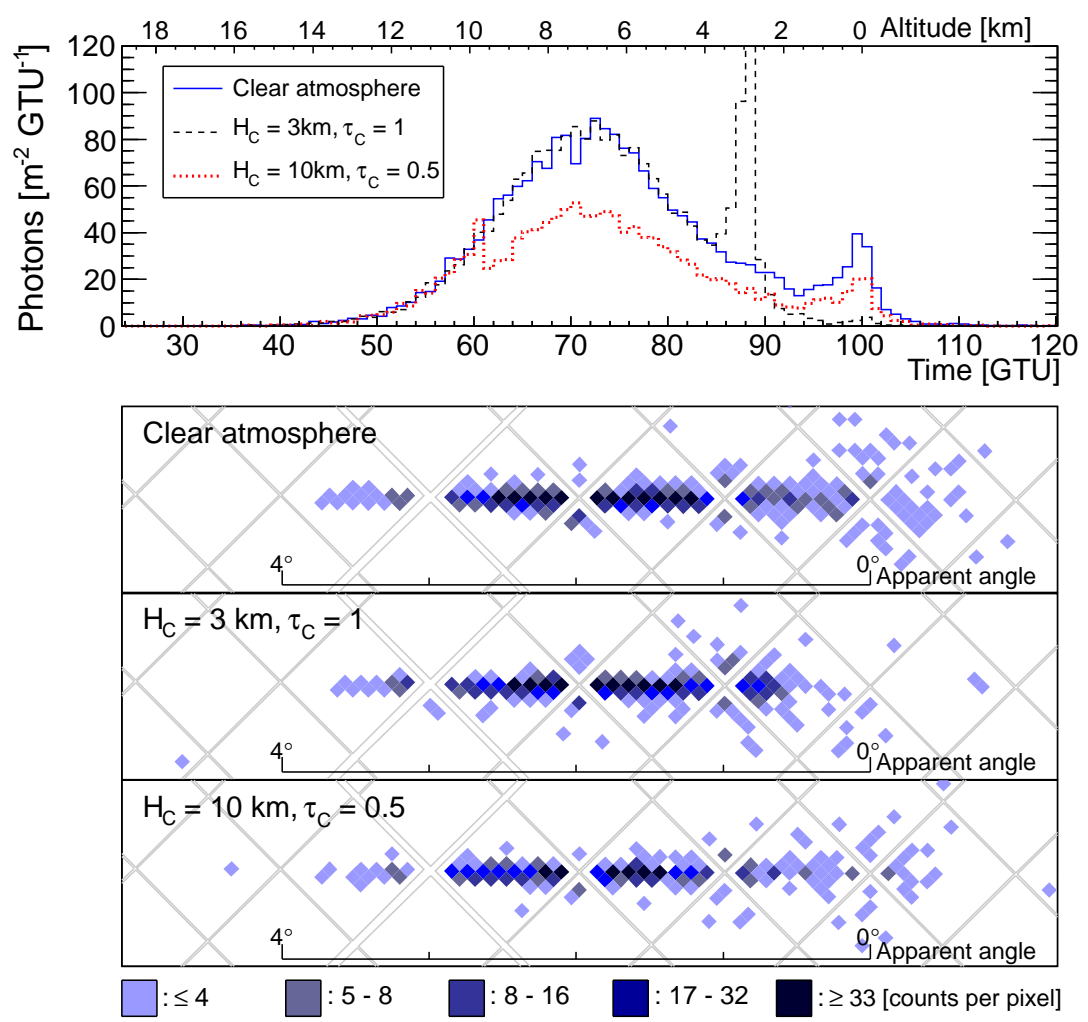

Fig. 2 Arrival time distribution of photons (top panel) from a proton induced EAS of $E_{0}=10^{20} \mathrm{eV}$ and $\Theta=60^{\circ}$ for different atmospheric conditions. The solid line represents the case for the clear atmosphere. Dashed and dotted lines denote the cloudy cases for $\tau_{\mathrm{C}}=1$ at $H_{\mathrm{C}}=3 \mathrm{~km}$ and $\tau_{\mathrm{C}}=0.5$ at $H_{\mathrm{C}}=10 \mathrm{~km}$, respectively. The axis on the top indicates the altitude where photons originate for the given arrival time. Bottom panels show the time-integrated images of signals on the FS detector for those three cases. The color scale indicates the number of signal counts per pixel. The horizontal position along the axis corresponds to the arrival time shown on the top panel. The gray lines indicate the boundaries of MAPMTs.

respectively. The horizontal axis is the absolute time. The time that the first shower particles reach the Earth's surface is set at 100 GTUs. The axis on the top indicates the altitude where photons originate for the given arrival time. Bottom panels display the time-integrated images of signals on the FS detector for those cases. The color scale indicates the number of signal counts per pixel. The horizontal position along the axis corresponds to the arrival time shown on the top panel.

In the clear atmosphere condition, the light curve indicates the EAS development, followed by the Cherenkov footprint on the surface. For EASs from $\Theta=60^{\circ}$ in this example, the apparent movement extends $\sim 2.5^{\circ}$ and lasts $\sim 50$ GTUs $(=125 \mu \mathrm{s})$. Using these observable data, the EAS parameters are reconstructed.

In case of the presence of clouds, EAS signals that appear are modified. If the optical depth of the cloud is large enough, the apparent shower track is effectively truncated. Upward photons scattered or emitted below the cloud are extinguished and 
Table 1 Average $\zeta(E)$ for different test clouds for $E>6.3 \times 10^{19} \mathrm{eV}$ with an assumed flux of $\propto E^{-3}[11]$.

\begin{tabular}{c|cccc}
\hline \multirow{2}{*}{ Cloud-top altitude $H_{\mathrm{C}}$} & \multicolumn{4}{|c}{ Optical depth $\tau_{\mathrm{C}}$} \\
\cline { 2 - 5 } & 0.05 & 0.5 & 1.5 & 5 \\
\hline $10 \mathrm{~km}$ & $88 \%$ & $66 \%$ & $37 \%$ & $18 \%$ \\
$7.5 \mathrm{~km}$ & $89 \%$ & $69 \%$ & $43 \%$ & $26 \%$ \\
$5 \mathrm{~km}$ & $88 \%$ & $82 \%$ & $74 \%$ & $70 \%$ \\
$2.5 \mathrm{~km}$ & $90 \%$ & $89 \%$ & $89 \%$ & $90 \%$ \\
\hline
\end{tabular}

do not contribute to the signals at the telescope. In this example, with a cloud at $3 \mathrm{~km}$, the apparent signals extend $\sim 2.5^{\circ}$ and last 40 GTUs. It is still feasible to apply the reconstruction techniques used in the case of the clear atmosphere by only using the measurements taken above the cloud.

As seen in the figure for the case of a small optical depth, photon signals that originated below the cloud are attenuated. This lowers the estimated energy of the EAS if the same techniques for the clear atmosphere are applied. Alternatively, the Cherenkov footprint is still observable and the orientation and apparent angular velocity are not affected, thus, the repercussion on arrival direction determination is limited.

To estimate the efficiency for EAS observation in cloudy conditions, we first define the 'geometrical aperture' that represents trigger aperture, assuming a single homogeneous atmosphere condition over the observation area. In practice, the geometrical aperture is determined by a number of EASs simulated over an area $S_{\text {sim }}$ far larger than that effectively observable by the telescope. For $N_{\text {trig }}$ triggering samples among $N_{\text {sim }}$ simulated EASs, the geometrical aperture is defined as follows:

$$
A \equiv \frac{N_{\text {trig }}}{N_{\text {sim }}} \cdot S_{\text {sim }} \cdot \Omega_{0},
$$

where $\Omega_{0}=\pi$ [sr] is the solid angle acceptance for $0^{\circ} \leq \Theta \leq 90^{\circ}$. In clear atmosphere condition, it reaches $\sim 4.4 \times 10^{5} \mathrm{~km}^{2}$ sr at $\sim 10^{21} \mathrm{eV}$ [11]. Then we define $\zeta$ as the ratio of geometrical aperture in cloudy conditions to that in the clear atmosphere condition. It is expressed as a function of energy by

$$
\zeta\left(E ; H_{\mathrm{C}}, \tau_{\mathrm{C}}\right)=\frac{A\left(E ; H_{\mathrm{C}}, \tau_{\mathrm{C}}\right)}{A_{0}(E)}
$$

where $A\left(E ; H_{\mathrm{C}}, \tau_{\mathrm{C}}\right)$ and $A_{0}(E)$ are geometrical apertures as a function of energy for the case with the test cloud and for clear atmosphere condition, respectively.

Table 1 summarizes the average $\zeta$ above $6.3 \times 10^{19} \mathrm{eV}$ for different test clouds [11]. The differential flux of EASs is assumed to be $\propto E^{-3}$.

For clouds at higher altitudes, the cases with large optical depths indicate significant suppression in the geometrical aperture. This is explained by a reduction of the photon flux at the main telescope.

In the case of clouds at lower altitudes, only a small portion of photons are affected. For $\Theta \gtrsim 25^{\circ}$, the maximum of the EAS development takes place above $\sim 3 \mathrm{~km}$ altitude. This particularly means that the case of the low-altitude cloud can be regarded as practically clear atmosphere for EASs from larger zenith angles. 
For clouds with optical depths such as $\tau_{\mathrm{C}}=0.05$, the reduction of signals is almost independent of cloud-top altitudes and its influence for trigger algorithms is negligibly small. In the case of clouds with $\tau_{\mathrm{C}}=0.5$, the signal reduction produced by the cloud is slightly dependent on the altitude. Naturally, the higher the cloud is, the more EAS light is absorbed, however, for trigger algorithms its influence is limited.

\section{Climatological average of cloud distribution}

In the following, we analyze existing satellite measurements from CALIPSO (Cloud Aerosol Lidar and Infrared Pathfinder Satellite Observations) [24] and compare them with the measurements from TOVS (TIROS Operational Vertical Sounder) [25]. As described in the previous section, the degree of cloud influence on the EAS observation depends greatly on the properties of clouds. It is, therefore, important to evaluate the cloud distribution over the geographical regions covered by the ISS orbit.

The NASA project TOVS, on-board NOAA's TIROS series of polar orbiting satellites, consists of three instruments: High-Resolution IR Sounder Modification 2; Stratospheric Sounding Unit; and Microwave Sounding Unit. These instruments had been designed to determine the radiance that is needed to calculate temperature and humidity profiles up to the stratosphere. These data have a good spectral distribution and provide the optical depth and altitude of clouds, applying their own radiative transport model. In the present work, we use data taken between 1988 and 1994.

CALIPSO forms a part of the A-Train Satellite Constellation [26], a group of satellites which carry out atmospheric measurements. CALIPSO consists of a twowavelength polarization-sensitive LIDAR, and two passive imagers operating in the visible and IR bands. Data from these instruments are used to determine the vertical distribution of clouds and aerosols, along with their optical and physical properties. CALIPSO performs a sun-synchronous orbit at an altitude of $705 \mathrm{~km}$ with a $98.2^{\circ}$ inclination. With a $60-\mathrm{m}$ vertical resolution measurement by CALIOP (CloudAerosol Lidar with Orthogonal Polarization) [27], CALIPSO's LIDAR, the extinction coefficients of the clouds $\alpha_{C}(h)$ are provided as a function of altitude up to $20.2 \mathrm{~km}$. The horizontal resolution is $5 \mathrm{~km}$ along the orbit. Cloud data from CALIOP are incorporated into the Imaging Infra-red Radiometer (IIR) retrieval algorithm [28].

To compare with the analysis of the TOVS data, the cloud optical depth $\tau_{\mathrm{C}}$ is determined by integrating $\alpha_{\mathrm{C}}(h)$ from $20.2-\mathrm{km}$ altitude to the surface boundary, namely either water or land. Since the LIDAR measurement can penetrate through the clouds, there is no unique definition for cloud top in the CALIPSO data. Therefore, for the CALIPSO analysis, we define the cloud-top altitude $H_{\mathrm{C}}$ as the altitude below which the optical depth exceeds 0.1, namely

$$
\int_{H_{\mathrm{C}}}^{20.2[\mathrm{~km}]} \alpha_{\mathrm{C}}(h) d h=0.1 .
$$

If $\tau_{\mathrm{C}}<0.1$, no cloud-top altitude is determined and the region under the scope is counted as clear atmosphere. 


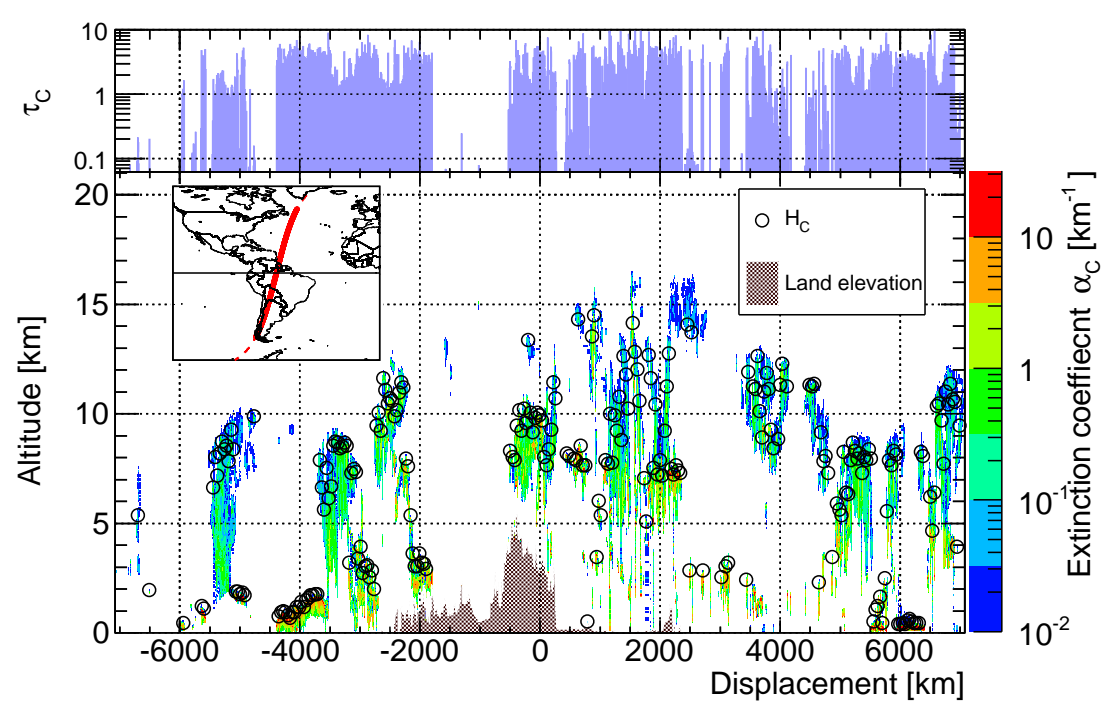

Fig. 3 An example of CALIPSO data for the $\alpha_{\mathrm{C}}$ profile in color scale on the coordinates of altitude versus elongated displacement along the orbit shown in the lower part. The shaded region represents the land elevation. $H_{\mathrm{C}}$ are indicated at every $50-\mathrm{km}$ displacement by circles. $\tau_{\mathrm{C}}$ are indicated in the upper part. The data were taken on May 1st, 2010, along a part of the orbit within $\pm 51.6^{\circ}$ latitudes shown by the bold curve on the inset map. The origin of the horizontal axis is at the Equator and positive values represents the North Hemisphere.

Fig. 3 demonstrates an example of the $\alpha_{\mathrm{C}}$ profile in color scale from CALIPSO. In the lower part of the figure, the coordinates are altitude versus northward displacement from the Equator along the orbit. $H_{\mathrm{C}}$ is also indicated at every $50-\mathrm{km}$ displacement by circles. The land elevation is represented by the shaded region. In the upper part, $\tau_{\mathrm{C}}$ is indicated by the histogram. The data were taken on May 1st, 2010 and are limited to within $\pm 51.6^{\circ}$ latitudes along the part of the orbit shown in the inset map.

In this example, one can see clouds in various regions with widely distributed cloud-top altitudes below $\sim 15 \mathrm{~km}$. Clear atmosphere regions are also observed around $-1500-\mathrm{km}$ - and $-6000-\mathrm{km}$ - displacements and several other places. There are also regions with relatively low cloud-top altitudes, for example around $-4000-\mathrm{km}$ displacement, where only the observation of near-vertical EASs are affected.

In the present work, we use a sample of the CALIPSO database selected over the year 2010, and apply the above calculations. By analyzing the databases mentioned above, the probability distribution functions $\mathscr{F}_{\mathrm{C}}\left(H_{\mathrm{C}}, \tau_{\mathrm{C}}\right)$ that give the relative occurrence of the cloud types are obtained. The climatological average of the clouds is inferred from these functions. To characterize the cloud, we first categorize clouds by their top altitudes $H_{\mathrm{C}}$ into four ranges of $<3.2 \mathrm{~km}, 3.2-6.5 \mathrm{~km}, 6.5-10 \mathrm{~km}$ and $>10 \mathrm{~km}$. In addition, the optical depths $\tau_{\mathrm{C}}$ are tabulated into four ranges of $<0.1$, $0.1-1,1-2$ and $>2$. In both databases, we only select the entries of nighttime measurements in the region within $\pm 51.6^{\circ}$ latitudes. 
Table 2 Relative occurrence of cloud categories over the ISS orbit, taken from the TOVS and CALIPSO presented as a matrix of cloud-top altitude versus optical depth. For CALIPSO analysis, the cases with $\tau_{\mathrm{C}}<0.1$ are all summed up as clear atmosphere. The analysis of TOVS is from Ref. [11].

\begin{tabular}{|c|c|c|c|c|c|c|c|c|}
\hline \multirow{3}{*}{ Cloud-top altitude $H_{\mathrm{C}}$} & Relat & e occul & nce $(\mathrm{TC}$ & & Relat & e occur & ice $(\mathrm{C}$ & IPSO) \\
\hline & \multicolumn{8}{|c|}{ Optical depth $\tau_{\mathrm{C}}$} \\
\hline & $<0.1$ & $0.1-1$ & $1-2$ & $>2$ & $<0.1$ & $0.1-1$ & $1-2$ & $>2$ \\
\hline$>10 \mathrm{~km}$ & $1.2 \%$ & $5.0 \%$ & $2.5 \%$ & $5.0 \%$ & \multirow{4}{*}{$38 \%$} & $4.7 \%$ & $4.7 \%$ & $4.7 \%$ \\
\hline $6.5-10 \mathrm{~km}$ & $<0.1 \%$ & $3.2 \%$ & $4.2 \%$ & $8.5 \%$ & & $4.5 \%$ & $4.8 \%$ & $6.0 \%$ \\
\hline $3.2-6.5 \mathrm{~km}$ & $<0.1 \%$ & $2.0 \%$ & $3.0 \%$ & $6.0 \%$ & & $3.2 \%$ & $1.7 \%$ & $6.4 \%$ \\
\hline$<3.2 \mathrm{~km}$ & $31 \%$ & $6.4 \%$ & $6.0 \%$ & $16 \%$ & & $2.8 \%$ & $0.9 \%$ & $17 \%$ \\
\hline
\end{tabular}

Table 3 Comparison of clouds occurrence results from TOVS and CALIPSO data. Types of cloudy conditions are assumed: (a) for low-cloud or $\tau_{\mathrm{C}}<0.1$, (c) for high-cloud with $\tau_{\mathrm{C}}>1$ and (b) for any other intermediate.

\begin{tabular}{|c|c|c|c|c|c|c|c|}
\hline \multirow{3}{*}{ Cloud-top altitude } & Relati & occurr & $\mathrm{e}(\mathrm{TO}$ & & Relative & currenc & ALIPSO) \\
\hline & \multicolumn{7}{|c|}{ Optical depth $\tau_{\mathrm{C}}$} \\
\hline & $<0.1$ & $0.1-1$ & $>1$ & Typ & $>1$ & $0.1-1$ & $<0.1$ \\
\hline \multirow{3}{*}{$\begin{array}{c}\mathrm{HC}\left(H_{\mathrm{C}}>6.5 \mathrm{~km}\right) \\
\mathrm{MC}\left(H_{\mathrm{C}}=3.2-6.5 \mathrm{~km}\right) \\
\mathrm{LC}\left(H_{\mathrm{C}}<3.2 \mathrm{~km}\right)\end{array}$} & \multirow[b]{3}{*}{$61 \%$} & \multirow{3}{*}{\multicolumn{2}{|c|}{$19 \%$}} & \multirow{3}{*}{$\begin{array}{l}\text { (c) } \\
\text { (b) } \\
\text { (a) }\end{array}$} & $20 \%$ & \multirow{3}{*}{$21 \%$} & \multirow[b]{3}{*}{$59 \%$} \\
\hline & & & & & & & \\
\hline & & & & & & & \\
\hline
\end{tabular}

In Table 2, the relative occurrence of cloud properties from analyses of TOVS and CALIPSO data are summarized on an $H_{\mathrm{C}}-\tau_{\mathrm{C}}$ matrix. As mentioned above, the clouds with $\tau_{\mathrm{C}}<0.1$ for CALIPSO are classified as clear atmosphere.

In Table 3, results from TOVS and CALIPSO data are compared. Following the meteorological convention [29], clouds are sorted by their top altitudes into lowcloud (LC; $H_{\mathrm{C}}<3.2 \mathrm{~km}$ ), middle-cloud (MC; $H_{\mathrm{C}}=3.2-6.5 \mathrm{~km}$ ), or high-cloud (HC; $H_{\mathrm{C}}>6.5 \mathrm{~km}$ ). In addition to optical depth, they are summarized by types (a), (b), and (c) as defined below. Dividing matrices in Table 2, we use three types: (a) for LC or $\tau_{\mathrm{C}}<0.1$, (c) for HC with $\tau_{\mathrm{C}}>1$ and (b) for other cases. The type (b) includes MC with $\tau_{\mathrm{C}}>0.1$ and, otherwise, ones with $\tau_{\mathrm{C}}=0.1-1$, excluding the LC cases.

First of all, the results from the two analyses are in good agreement. The influence of clouds at higher altitudes and/or with larger optical depths is more significant to the EAS observation. 'Optically thick' high-clouds may especially reduce the efficiency of EAS observation, as can also be seen in Table 1. This corresponds to type (c). Note that this effect does not apply to the EAS from large zenith angles. For the intermediate type (b), the detection of such clouds is relevant so that EASs detected under such conditions are not confused with those under the type (a). On the other hand, in the type (a) case, low-clouds for most of the EASs may act as a clear atmosphere that do not hide the brightest part of EAS development. In this case, the cloud-top altitude within FOV of the main telescope is determined by the IR camera measurement to discriminate the cloud-free interval of light curves as seen in Fig. 2.

Apart from the average occurrence of clouds, the global distribution and seasonal dependence are also relevant in space-based observation. They result from a complex system of geographical, eg. land versus ocean, meteorological, and other factors (see Ref. [11,29] for discussion). We investigate the TOVS database, covering a period of 7 years, in all possible locations for JEM-EUSO. The nighttime duration is $34 \%$ on 


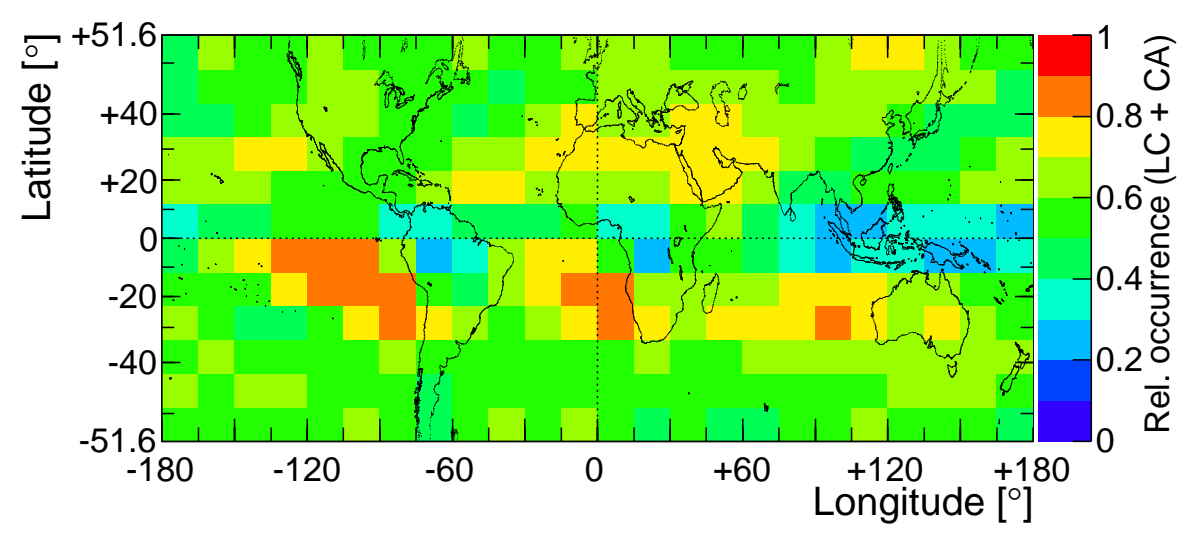

Fig. 4 Global distribution of occurrence of the sum of low-cloud and clear atmosphere from TOVS data in color scale. The projection reproduces a constant residence time of the ISS in each bin.

Table 4 Average relative occurrence of different cloud types by three-month seasons of year. The sum of types (a) plus (b), and the case of type (a) alone are summarized for each Earth's hemisphere.

\begin{tabular}{|c|c|c|c|c|c|}
\hline \multirow{2}{*}{ Type } & \multirow{2}{*}{ Hemisphere } & \multicolumn{4}{|c|}{ Month of year (March, April, .., February) } \\
\hline & & $\mathrm{M}$ & $\mathrm{J} \quad \mathrm{A}$ & $\mathrm{S} \quad \mathrm{O} \quad \mathrm{N}$ & D J \\
\hline$(a$ & $\begin{array}{l}\text { North } \\
\text { So }\end{array}$ & $\begin{array}{ll}81 \% & \\
& 79 \%\end{array}$ & $\begin{array}{l}76 \% \\
83 \%\end{array}$ & $\begin{array}{l}79 \% \\
82 \%\end{array}$ & $\begin{array}{l}82 \% \\
72 \%\end{array}$ \\
\hline$(2$ & $\begin{array}{l}\text { North } \\
\text { South }\end{array}$ & $\begin{array}{ll}59 \% & \\
& 60 \%\end{array}$ & $\begin{array}{l}56 \% \\
65 \%\end{array}$ & $\begin{array}{l}59 \% \\
64 \%\end{array}$ & $60 \%$ $58 \%$ \\
\hline
\end{tabular}

average over the ISS orbit, largely depending on latitudes due to the different twilight durations. To reduce such uncertainties, all data, including daytime, are analyzed.

Fig. 4 indicates the global map of the occurrence of low-cloud (LC) plus clear atmosphere (CA) in color scale, averaging all the data. The projection of the map reproduces a constant residence time of the ISS in each bin.

As previously mentioned, such conditions do not or only slightly affect the apparent signals of EASs. Therefore, a high occurrence of these conditions is advantageous for EAS observations. In addition to the argument in Table 3, the global average is $61 \%$ for the occurrence of favorable conditions. It is worthwhile to mention that there are regions with distinctly low and high occurrences. The former regions are found in land around equatorial zones that coincide with tropical rainforest climate in Köppen classification [30]. The latter widely appear above oceans, especially in the South Hemisphere. Relatively high occurrences of favorable condition are also seen in the regions of desert climate in North Africa, Middle East, and Australia.

Table 4 shows the average relative occurrence of different cloud types as a function of season of year. The sum of types (a) plus (b), and the case of type (a) alone are summarized as three-month average for each Earth's hemisphere.

In general, the seasonal variation in every test case is a small effect with an order of $\pm 5 \%$ of the average. The difference in the average between hemispheres is marginal, while in both hemispheres, winter tends to have higher occurrence than 
summer. The altitude where clouds are formed depends on temperature. The fiducial volume for EAS observation thus increases in the winter as the cloud-top altitudes descend. Note that the data used in this analysis also contain the daytime measurements. For the daytime, the cloud coverage is similar to that for nighttime [11,29]. Note that the temperatures are higher and hence cloud altitudes are also higher. The result herein thus constitutes a conservative estimation of the occurrence of favorable condition for EAS observations from space.

\section{Overall efficiency of EAS observation}

The overall exposure in the JEM-EUSO mission obviously suffers from the presence of clouds. Such an impact is estimated as a ratio for the average effective aperture to the geometrical aperture for clear atmosphere. This is expressed as the convolution of the trigger efficiency and the occurrence of assumed cloud properties in the present work. Using the already defined function $\zeta$ weighted by $\mathscr{F}$, the average ratio $\kappa_{\mathrm{C}}^{\prime}$ in aperture to that in clear atmosphere $A_{0}(E)$ is written as follows:

$$
\kappa_{\mathrm{C}}^{\prime}(E)=\int_{0}^{H_{0}} \int_{0}^{\infty} \zeta\left(E ; H_{\mathrm{C}}, \tau_{\mathrm{C}}\right) \cdot \mathscr{F}\left(H_{\mathrm{C}}, \tau_{\mathrm{C}}\right) d \tau_{\mathrm{C}} d H_{\mathrm{C}} .
$$

After the EASs have triggered the detector, the reconstruction of these events follows. To achieve reasonable accuracies, we impose a minimal requirement: the visibility of the EAS maximum. We require that the EAS reaches its maximum above the cloud-top altitude or when the cloud has $\tau_{\mathrm{C}}<1$. The latter case includes clouds of the type (b) in our classification. In such situations, estimations of energy and determination of maximum position suffer from the distorted light curve. Therefore, the observed EAS events should be carefully treated. There may be cases that requires these events to be eliminated in scientific analysis. However, in addition to the type (a) case, these events can still be used for analysis of arrival direction that does not need the highest quality of EAS data. In both cases, enough information from signals above and through the cloud is obtained since the arrival direction determination is simply based on unchanged apparent angular velocity of EASs.

Taking this requirement into account, Eq. (4) is revised as follows:

$$
\begin{gathered}
\kappa_{\mathrm{C}}(E)=\frac{1}{A_{0}(E)} \\
\cdot \int_{0}^{H_{0}}\left[\int_{1}^{\infty} A\left(E, H_{\mathrm{C}}, \tau_{\mathrm{C}} \mid H_{\mathrm{C}}<H_{\max }\right) \cdot \mathscr{F}\left(H_{\mathrm{C}}, \tau_{\mathrm{C}}\right) d \tau_{\mathrm{C}}\right. \\
\left.+\int_{0}^{1} A\left(E, H_{\mathrm{C}}, \tau_{\mathrm{C}}\right) \cdot \mathscr{F}\left(H_{\mathrm{C}}, \tau_{\mathrm{C}}\right) d \tau_{\mathrm{C}}\right] d H_{\mathrm{C}}
\end{gathered}
$$

where $H_{\max }$ is the altitude of the EAS development maximum. In the present analysis, TOVS data is used to estimate $\mathscr{F}\left(H_{\mathrm{C}}, \tau_{\mathrm{C}}\right)$.

In Fig. 5, the relation between $\kappa_{C}^{\prime}$ and energy is shown by triangles and $\kappa_{C}$ is plotted by closed circles [11]. The error bar denotes an estimated uncertainty on the points, mainly due to the cloud coverage data.

Including cloudy condition, $\kappa_{\mathrm{C}}^{\prime}$ is $80 \%$ or higher at energies of interest. It increases with energy. Around $10^{21} \mathrm{eV}$, the trigger aperture is nearly the same as that in clear 


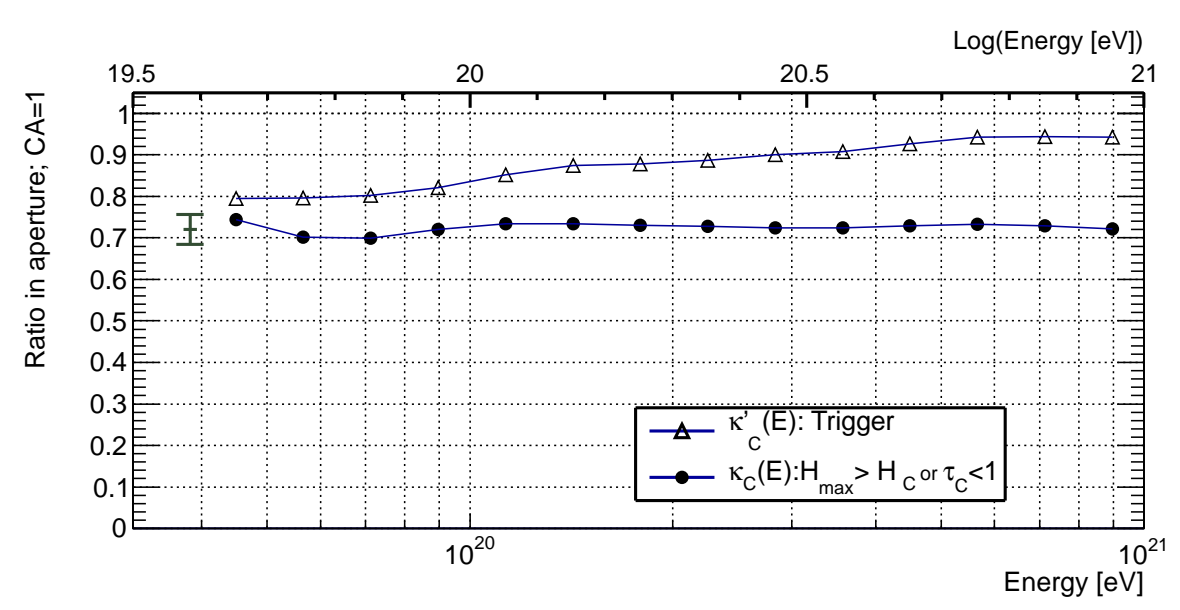

Fig. 5 Ratio of the geometrical aperture for averaged cloudy condition to that from clear atmosphere as a function of energy [11]. The triangles and circles show $\kappa_{C}^{\prime}$ and $\kappa_{C}$ defined in Eqs. (4) and (5), respectively. In the latter case, $H_{\mathrm{C}}<H_{\max }$ or $\tau_{\mathrm{C}}<1$ are required for triggering EAS events. The error bar denotes an estimated uncertainty on $\kappa_{\mathrm{C}}$.

atmosphere. At such energies, a large number of photons still reaches the telescope to trigger it despite the extinction loss in the cloud. The reference aperture $A_{0}(E)$ for clear atmosphere condition can be found in Ref. [11,31].

If the criterion of visibility of the EAS maximum is applied, the corresponding efficiency $\kappa_{\mathrm{C}}$ is almost constant $\sim 72 \%$. The independence of energy is limited due to the fact that $X_{\max }$, the atmospheric depth at EAS maximum, does not vary much within the concerned energy range [32], while $H_{\max }$ increases with zenith angles. For EASs from proton with $E=10^{20} \mathrm{eV}, H_{\max }$ is $\sim 3 \mathrm{~km}, \sim 7 \mathrm{~km}$ and $\sim 11 \mathrm{~km}$ for $\Theta=$ $30^{\circ}, 60^{\circ}$ and $75^{\circ}$, respectively. In most zenith angles, it is higher than typical cloudtop altitudes during nighttime as seen in Tables 2 and 3. This criterion ensures that the apparent EAS profile does not introduce significant distortion to fitting of the EAS profile. It is worthwhile to mention that our results seem dependent on combinations of hadronic interaction models and primary particles. However, $\kappa^{\prime}$ only varies by $\sim \pm 4 \%$, changing $H_{\max }$ by $1 \mathrm{~km}$ for the TOVS data. Note that $1-\mathrm{km}$ difference in altitude is equivalent to typical $X_{\max }$ dependence among those combinations.

In Ref. [11,31], $\kappa_{C}(E)$ is referred to as the 'cloud efficiency'. It is an important factor for estimating the effective exposure of the JEM-EUSO mission. Detailed studies about the reconstruction in clear atmosphere condition are described in Refs. [18-20]. It should be emphasized that the information retrieved by the AM system may be of use to eliminate the low quality region in FOV based on local cloud properties $[8,15]$. Further study on reconstruction in cloudy conditions is in progress. 


\section{Summary and discussion}

In the present article, we give an overview of the EAS observation technique in cloudy conditions for the JEM-EUSO mission. We focus on the following aspects: the influence of cloud presence on space-based EAS observation; the distribution of the clouds sorted with their properties, as well as, geographical and seasonal dependence; and the estimation of the overall observation efficiency.

For the space-based observation, the influence of the clouds varies with cloud-top altitude and optical depth. It also depends on the zenith angle of the EAS, relating to the altitude of development. From EAS simulation studies with commonly accepted interaction models [32], the difference of $X_{\max }$ at $E=10^{20} \mathrm{eV}$ is $\sim 100 \mathrm{~g} \mathrm{~cm}^{-2}$ between proton and iron induced EASs. This means that the latter reach maximum development at $\sim 1-\mathrm{km}$ higher than the former. The influence of cloud presence is weaker for this case. Thus, the simulation studies with proton primaries, therefore, constitutes a conservative performance estimation.

Low-clouds only affect the final stage of EAS development. The light curve still allows energy and $X_{\max }$ to be reconstructed since the relevant part of the development is observable without distortion. The arrival direction of UHECRs is determined by means of the same approach used for clear atmosphere condition, as well. For low clouds with substantial optical depth, the AM system will locate them, along with their top altitude distribution $[8,15]$. Utilizing these additional pieces of information, the Cherenkov footprint gives far better determination on the impact position on the cloud. Though it is not studied in detail, we wish to mention that such clouds located in mildly light-polluted urban areas may play a positive role in blocking the anthropogenic light and, therefore, allowing for EAS measurement, as well.

High-clouds, with relatively small optical depths, only slightly attenuate the photons from the EAS. In this case, the information on the EAS tracks with its temporal development is obtained with little or no disturbance. This allows for the EAS events obtained in such condition to be used for arrival direction distribution analysis. The estimated energy is potentially affected, seen as if the EAS is of a lower energy. For those atmospheric conditions, the importance of atmospheric monitoring is more pronounced. To tag these kinds of events, the region in the FOV with such an atmospheric condition are identified in the AM system within its sensitivity [8].

The overall influence of the clouds is more dependent on their climatological properties. The analyses of TOVS and CALIPSO databases show consistent distributions of clouds sorted by the expected degrees of influence to EAS observation. The average cloud properties from the TOVS database studied in Ref. [11], is found to be in good agreement with the result from the CALIPSO database. Referring to the TOVS result, the occurrence of clear atmosphere is $32 \%$. One can assume that this case guarantees good condition for both ground-based and spaced-based observations. Moreover, for the space-based observation, the cumulative occurrence increases up to $61 \%$ by adding the low-cloud cases. On these conditions, the observed events may be used for arrival direction, spectrum and $X_{\max }$ analyses, meeting observational requirements of the mission [2]. Another $20 \%$ case of the cloudy condition still allows a significant fraction of signals from the EAS to reach the JEMEUSO telescope. Using information of the AM system, triggered events observed un- 
der such circumstances are clearly labeled to discriminate from those with the above mentioned good condition.

In this case for each observed EAS event, arrival direction is only little affected, despite the uncertainty by the extinction loss in the cloud with weakly constrained optical depth. On the other hand, a likely distorted light curve prevents precise determination of energy and $X_{\max }$. By determining the lower bound of the primary energy estimated by the amount of signals from EAS, these events may be used for scientific analysis that does not require the best quality of the data.

In the present work, we evaluate the global distribution with TOVS. The result shows some locality that is explained by conventional knowledge on the climate. The annual variation is only found at an order of a few percent. We also note that the annual variation acts as a factor in exposure distribution on Celestial Sphere [11, 31]. As a convolution of the cloud population and the observation efficiency, the aperture at energies of interest is $80 \%$ and higher in comparison with that in the clear atmosphere condition. Taking into account the visibility of the EAS maximum, the overall cloud efficiency $\kappa_{\mathrm{C}}$ is evaluated to be $72 \%$. This factor is one of the key parameters in expected exposure evaluation (see Ref. [11,31]).

It should be mentioned that simulation studies in Refs $[3,33]$ showed the feasibility of reconstructing EAS with reasonable accuracy in the presence of clouds. For a given energy, the apparent length of EAS signals mainly depends on the zenith angle. The quality of reconstruction for events truncated by a cloud may be comparable to the case with a smaller zenith angle in clear atmosphere (see Refs. [18-20]). In addition to the data measured from the AM system $[8,15]$, meteorological information from ground stations satellites, and global models are also available for the FOV of the JEM-EUSO telescope at any given time. Further studies are in progress towards the development of a data analysis scheme, including all available information from the main telescope, the AM system and other data regarding atmospheric conditions.

\section{Acknowledgments}

The present work has been supported in part by the European Space Agency Topical Team activities; for German consortium by Deutsches Zentrum für Luft- und Raumfahrt; the Helmholtz Alliance for Astroparticle Physics funded by the Initiative and Networking Fund of the Helmholtz Association, and for Spanish consortium by Ministerio de Ciencia e Innovación under projects AYA2009-06037-E/ESP, AYAESP 2010-19082, AYA2011-29489-C03-01, AYA2012-39115-C03-01 and CSD200900064 (Consolider MULTIDARK) and Comunidad de Madrid under project S2009/ ESP-1496. CALIPSO data used in the present work were obtained from the NASA Langley Research Center Atmospheric Science Data Center. The authors wish to thank anonymous reviewers for valuable comments to improve the manuscript. 


\section{References}

1. Santangelo, A.; Picozza, P.; Ebisuzaki, T. et al. (JEM-EUSO Collaboration), "Status of the JEM-EUSO Mission", Proceedings of 33rd International Cosmic Ray Conference, Rio de Janeiro, ID0738 (2013).

2. Adams, J.H. Jr. ; Ahmad, S.; Albert, J.-N. et al. (JEM-EUSO Collaboration), "The JEM-EUSO mission (An introduction to the focused issue)", in this issue.

3. Takahashi, Y. and the JEM-EUSO Collaboration, "The JEM-EUSO Mission", New Journal of Physics 11, 065009/1-21 (2009).

4. F. Kajino; Casolino, M.; Ebisuzaki, T. et al. (JEM-EUSO Collaboration), "The JEM-EUSO Instruments", Proceedings of 33rd International Cosmic Ray Conference, Rio de Janeiro, ID1128 (2013).

5. Adams, J.H. Jr.; Ahmad, S.; Albert, J.-N. et al. (JEM-EUSO Collaboration), "An overview of the JEMEUSO instrument", in this issue.

6. For review, Letessier-Selvon, A.; Stanev, T., "Ultrahigh energy cosmic rays", Review of Modern Physics 83, 907-942 (2011).

7. Neronov, A.; Rodriguez Frías, M.D.; Toscano, S. et al. (JEM-EUSO Collaboration), "Atmospheric Monitoring system of the JEM-EUSO telescope", Proceedings of 33rd International Cosmic Ray Conference, Rio de Janeiro, ID1072 (2013).

8. Adams, J.H. Jr.; Ahmad, S.; Albert, J.-N. et al. (JEM-EUSO Collaboration), "The AM system of the JEM-EUSO instrument", in this volume.

9. NASA, "ISS User's Guide-Release 2.0" (2000).

10. JAXA Human Space Systems and Utilization Program Group, "Kibo Handbook" (2007).

11. Adams, J.H. Jr. ; Ahmad, S.; Albert, J.-N. et al., "An evaluation of the exposure in nadir observation of the JEM-EUSO mission", Astroparticle Physics 44, 76-90 (2013).

12. Adams, J.H. Jr. ; Ahmad, S.; Albert, J.-N. et al. (JEM-EUSO Collaboration), "The Photodetector Module of the JEM-EUSO mission", in this issue.

13. Catalano, O.; Bertaina, M.; Casolino, M. et al. (the JEM-EUSO Collaboration), "The trigger system of JEM-EUSO Telescope" Proceedings of 31st International Cosmic Ray Conference, Lodz, \#0326 (2009).

14. Rodriguez Frias, M.D.; Licandro, J.; Sabau, M.D. et al. (JEM-EUSO Collaboration), "Towards the Preliminary Design Review of the Infra Camera of the JEM-EUSO Space Mission", Proceedings of 33rd International Cosmic Ray Conference, Rio de Janeiro, ID0900 (2013).

15. Adams, J.H. Jr. ; Ahmad, S.; Albert, J.-N. et al. (JEM-EUSO Collaboration), "The Infrared Camera onboard JEM-EUSO", in this issue.

16. Toscano, S.; Valore, F.; Neronov, A. et al. (JEM-EUSO Collaboration), "An End to End Simulation code for the IR-Camera of the JEM-EUSO Space Observatory", Proceedings of 33rd International Cosmic Ray Conference, Rio de Janeiro, ID0514 (2013).

17. Toscano, S.; Valore, F.; Neronov, A. et al. (JEM-EUSO Collaboration), "LIDAR treatment inside the ESAF Simulation Framework for the JEM-EUSO mission”, Proceedings of 33rd International Cosmic Ray Conference, Rio de Janeiro, ID0530 (2013).

18. Mernik, T.; Guzman, A.; Fenu, F. et al. (JEM-EUSO Collaboration), "Simulating the JEM-EUSO Mission: Expected Reconstruction Performance", Proceedings of 33rd International Cosmic Ray Conference, Rio de Janeiro, ID1072 (2013).

19. Adams, J.H. Jr. ; Ahmad, S.; Albert, J.-N. et al. (JEM-EUSO Collaboration), "Performances of JEMEUSO: energy and $X_{\max }$ reconstruction", in this issue.;

20. Adams, J.H. Jr. ; Ahmad, S.; Albert, J.-N. et al. (JEM-EUSO Collaboration), "Performances of JEMEUSO: angular reconstruction", in this issue.

21. Berat, C.; Bottai, S.; De Marco, D. et al., "ESAF: Full Simulation of Space-Based Extensive Air Showers Detectors", Astroparticle Physics 33, 211-247 (2010).

22. Deirmendjian, D., "Electromagnetic Scattering on Spherical Polydispresions", American Elsevier Publishing Company Inc., New York (1969).

23. Liou, K.N., "Radiation and Cloud Processes in the Atmosphere", Oxford University Press (1992).

24. CALIPSO, http://www.nasa.gov/mission_pages/calipso/main/

25. TOVS, http://www.ozonelayer.noaa.gov/action/tovs.htm/

26. A-Train Constellation, http://http://atrain.nasa.gov/

27. CALIOP, http://www-calipso.larc.nasa.gov/about/payload.php\#CALIOP

28. Garnier, A; Pelon, J.; Dubuission, P. et al., "Retrieval of Cloud Properties Using CALIPSO Imaging Infrared Radiometer. Part I: Effective Emissivity and Optical Depth", Journal of Applied Meteorology and Climatology 51, 1407-1425 (2012).

29. Garino, F., Master Thesis, University of Torino (2011) and references therein. 
30. Eg. Peel, M.C.; Finlayson, B.L. and McMahon, T.A., "Updated world map of the Köppen-Geiger climate classification", Hydrology and Earth System Sciences 11, 1633-1644 (2007).

31. Adams, J.H. Jr. ; Ahmad, S.; Albert, J.-N. et al. (JEM-EUSO Collaboration), "The JEM-EUSO observational technique and exposure", in this issue.

32. Roh, S.; Kim, J; Ryu, D. et al., "A comparison study of CORSIKA and COSMOS simulations for extensive air showers", Astroparticle Physics 44, 1-8 (2013).

33. Abu-Zayyad, T.; Jui, C.C.H. and Loh, E.C., "The effect of clouds on air showers observation from space", Astroparticle Physics 21, 163-182 (2004). 